\title{
Rapid evolution of the Helicobacter pylori AlpA adhesin in a high gastric cancer risk region from Colombia
}

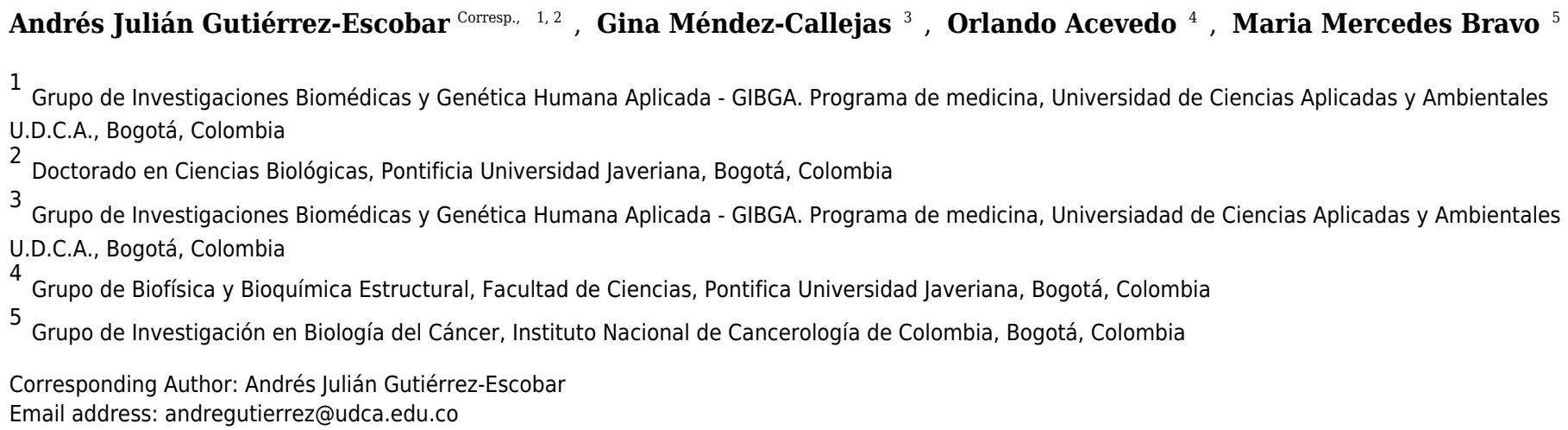

To be able to survive, H. pylori must adhere to the gastric epithelial cells of its human host. For this purpose, the bacterium employs an array of adhesins, for example, AlpA. The adhesin AlpA has been proposed as a major adhesin because of its critical role in human stomach colonization. Therefore, understanding how AlpA evolved could be important for the development of new diagnostic strategies. However, the genetic variation and microevolutionary patterns of alpA have not been described in Colombia. The study aim was to describe the variation patterns and microevolutionary process of alpA in Colombian clinical isolates of $H$. pylori. The existing polymorphisms, which are deviations from the neutral model of molecular evolution, and the genetic differentiation of the alpA gene from Colombian clinical isolates of $H$. pylori were determined. The analysis shows that gene conversion and purifying selection have shaped the evolution of three different variants of alpA in Colombia. 
1 Rapid evolution of the Helicobacter pylori AlpA adhesin in a 2 high cancer gastric risk region from Colombia

3

4 Andrés Julián Gutiérrez-Escobar ${ }^{1 *}$, Gina Méndez-Callejass ${ }^{1}$, Orlando Acevedo², María Mercedes Bravo ${ }^{3}$

5

61 Grupo de Investigaciones Biomédicas y Genética Humana Aplicada - GIBGA. Programa de medicina.,

7 Universidad de Ciencias Aplicadas y Ambientales U.D.C.A., Bogotá, Colombia.

8 * Doctorado en Ciencias Biológicas, Pontificia Universidad Javeriana, Bogotá, Colombia.

92 Grupo de Biofísica y Bioquímica Estructural, Facultad de Ciencias, Pontificia Universidad Javeriana,

10 Bogotá, Colombia.

113 Grupo de Investigación en Biología del Cáncer, Instituto Nacional de Cancerología, Bogotá, Colombia.

Corresponding Author:

14

Email address: andresjulian1981@gmail.com

15 


\section{Abstract}

To be able to survive, $H$. pylori must adhere to the gastric epithelial cells of its human host. For this purpose, the bacterium employs an array of adhesins, for example, AlpA. The adhesin AlpA has been proposed as a major adhesin because of its critical role in human stomach colonization. Therefore, understanding how AlpA evolved could be important for the development of new diagnostic strategies. However, the genetic variation and microevolutionary patterns of alp $A$ have not been described in Colombia. The study aim was to describe the variation patterns and microevolutionary process of alp $A$ in Colombian clinical isolates of $H$. pylori. The existing polymorphisms, which are deviations from the neutral model of molecular evolution, and the genetic differentiation of the alp $A$ gene from Colombian clinical isolates of $H$. pylori were determined. The analysis shows that gene conversion and purifying selection have shaped the evolution of three different variants of alp $A$ in Colombia.

\section{Introduction}

H. pylori, a Gram-negative bacterium, has persistently colonized the stomach of half of the human population (Pérez - Pérez GI et al., 2004; Khalifa MM et al., 2010). This infection produces an asymptomatic inflammation of the gastric epithelium, but in some patients, it progresses toward a more severe clinical disease, such as ulcers and gastric cancer (Yakirevich E, Resnick MB, 2013).

Gastric cancer is the fifth most common cancer worldwide (Jemal et al., 2010; Bertuccio et al., 2009; Forman et al., 2014), and it is the second leading cause of cancer deaths (Ferlay et al., 2013); an infection with $H$. pylori is the strongest factor risk for its development (Helicobacter and Cancer Collaborative Group, 2001). In Colombia, the prevalence of this infection is universally high (Matta et al., 2017). However, the gastric cancer risk increases along with the altitudinal gradient (Torres et al., 2013). Thus, it is higher in the Andes region than along the Pacific coast (Kodaman et al., 2014); this phenomenon has been called the Colombian enigma (Correa \& Piazuelo, 2010).

The bacterium has coevolved with its human host for a period of 60,000 years, since the first major migratory wave outside Africa (Linz B et al., 2007). The bacterium has followed a similar dispersal pattern as its human host. Currently, seven populations of $H$. pylori have been identified: hpEurope, hpNEAfrica, hpAfrica1, hpAfrica2, hpAsia2, hpSahul and hpEastAsia (Yamaoka Y, 2009; Falush D et al., 2003; Achtman M et al., 1999; Moodley Y et al., 2009; Devi SM et al., 2007). A recent human migratory event was the colonization of the Americas, 525 years ago. During this colonization, new pathogens arrived to the American continent, including new strains of $H$. pylori, which caused the disappearance of $80 \%$ of the native population (Bianchine PJ, Russo TA, 1992; Parrish CR et al., 2008). It has been reported that H. pylori has followed unique evolutionary pathways in Latin-America (Gutiérrez-Escobar AJ et al., 2017; Muñoz-Ramírez ZY et al., 2017) and that the strains followed rapid adaptive processes in different countries of the region (Thorell K et al., 2017), establishing local independent lineages.

The bacterium has virulence factors that correlate with the risk of developing gastric diseases (Parsonnet J et al., 1997; Nomura AMY et al., 2002; Mahdavi J et al., 2002; Yamaoka Y et al., 
90

2002). H. pylori attach to the gastric epithelium thorough adhesins that contribute to the initial steps of the infection (Matsuo Y, Kido Y, 2017). AlpA ( 56 kDa) is an adhesin that is encoded by the locus alpAB (Alm R.A et al., 2000), which is essential for adherence to the human gastric epithelium (Odenbreit $\mathrm{S}$ et al., 1999). This adhesin is expressed by all clinical isolates (Odenbreit S, 2009) and is recognized in sera from infected patients (Xue J, 2005), and in addition, it induces the secretion of interleukin-8 (IL-8) (Lu H et al., 2007). AlpA represents an emerging virulence factor of $H$. pylori that has been gaining attention because of its potential as a vaccine target (Sun Z.L et al., 2010).

The aim of the present study was to describe the genetic diversity and microevolution of the adhesin AlpA at the population level in the high gastric cancer risk region of Colombia. Population genetics statistics and phylogenetic methods were performed to compare $H$. pylori strains from Colombian clinical isolates against strains from different geographical backgrounds.

\section{Materials and methods}

\subsection{DNA and protein sequences}

The DNA (alpA) and protein sequences (AlpA) were obtained from 115 genomes from the $H$. pylori stock collection, which was previously sequenced by our research group at the Instituto Nacional de Cancerología in Bogotá. The isolates belonged to patients with different types of gastric pathologies associated with $H$. pylori infection, as follows: 30 cases of Gastritis (G), 20 of Gastric Adenocarcinoma (GA), 28 of Atrophic Gastritis (AG), 30 of Intestinal Metaplasia, 5 of Gastritis concomitant with Duodenal Ulcer (G-DU) and 2 of Intestinal Metaplasia concomitant with Duodenal Ulcer (IM-DU) (Gutiérrez-Escobar AJ et al.,2017).

The reference pool sequences were obtained from $34 \mathrm{H}$. pylori strains, as follows: HspAmerind: Cuz20, PeCan4, Puno135, Sat464, Shi112, Shi169, Shi417, Shi470, v225d; HpEurope: 26695, B8, G27, HPAG1, ELS37, Lithuania75, SJM180; HspAsia: F57, XZ274, 51, 52, 35A, F32, F16, F30, 83; HspAsia2: SNT49, India7; HspWestAfrica: J99, 908, PeCan18, 2017, 2018, Gambia94/24 and HpSouthAfrica: SouthAfrica7.

\subsection{Phylogenetic analysis of alpA}

A total of 142 protein sequences for AlpA (108 sequences from Colombian isolates and 34 from reference) were aligned using the software Muscle V 3.8.31 (Edgar RC, 2004); the evolutionary model and the phylogenetic reconstruction was determined using MEGA V 7 (Kumar S et al., 2016) and the NJ algorithm (Saitou N, Nei M, 1987) with 1000 bootstrap repetitions for statistical robustness.

\subsection{Genetic diversity, natural selection tests and differentiation analysis of alp $A$}

The following population statistics: number of haplotypes $(\mathrm{H})$, haplotype diversity $(\mathrm{Hd})$, nucleotide diversity $(\mathrm{Pi})$, average number of nucleotide differences $(\mathrm{k})$, Theta estimator $(\theta \mathrm{w})$ and recombination events $(\mathrm{Rm})$ analyses were performed using DnaSP v 5.10 (Librado P, Rozas J, 2009). Deviations of the neutral model of molecular evolution were tested using the Tajima test 
128

(Tajima F, 1989) and the Z-test in which the average number of synonymous substitutions per synonymous site (dS) and the average number of non-synonymous substitutions per nonsynonymous site $(\mathrm{dN})$ were calculated using the modified Nei-Gojobori method with the JunkesCantor correction. The variance of the difference was computed using the bootstrap method (1000 replicates) using Mega v 7 (Kumar S et al., 2016). Finally, a sliding window analysis was applied to detect the evolutionary rate $\omega=\mathrm{dN} / \mathrm{dS}$ throughout the gene to identify specific regions under natural selection using the software DnaSP v 5.10 (Librado P, Rozas J, 2009).

The DNA sequences of Colombian isolates were grouped according to the gastric pathology of the patients: Gastritis (G), Gastric Adenocarcinoma (GA), Atrophic Gastritis (AG), Intestinal Metaplasia (IM), Gastritis concomitant with Duodenal Ulcer (GDU) and Intestinal Metaplasia concomitant with Duodenal Ulcer (IM-DU); the reference sequences were grouped according to their geographic origin. To detect the genetic heterogeneity and genetic flow, the following tests were applied: Hst, Kst, Kst*, Z, Z*. The HBK, Snn and chi squared tests were performed using the haplotype frequencies under the permutation of 1000 repetitions, as well as the tests for the haplotype diversity of Gst, Nst, Fst, and Da, and the gene flow (Nm), a measure of the genetic interaction, was estimated from FsT (Slatkin, 1985, 1987) using the software DnaSP v 5.10 (Librado P, Rozas J, 2009).

\subsection{Gene conversion analysis of alpA}

To test whether gene conversion generates genetic diversity in the alpA, the Betran's method (Betran E et al., 1997) implemented in the DnaSP v 5.10 software was used to compare Colombian isolates and the reference pool populations. RDP3 v 3.4 software was used to test gene conversion in the overall population using the GENECONV algorithm (Sawyer S, 1989). Only conversion tracks with $\mathrm{p}<0.05$ were considered.

\subsection{Type I functional divergence and site specific positive selection analyses}

The 3D structure of the AlpA protein was predicted using the server I-TASSER (Yang $\mathrm{J}$ et al., 2015) and tested using the MetaMQAPII server (Pawlowski M et al., 2008). DIVERGE V 3 software (Gu X et al., 2013) was used to estimate type-I functional divergence, which detects functional changes in a protein based on site-specific shifts in the evolutionary rates $(\mathrm{Gu} \mathrm{X}$, 1999). The software tested whether a significant change in the evolution rate has occurred by calculating the coefficient of divergence $(\theta \mathrm{D})$. Positive and negative selection was evaluated as the proportions of synonymous to non-synonymous substitution rates. The DNA sequences were aligned using the software Muscle V 3.8.31 (Edgar RC, 2004), and the alignment file was preprocessing by screening for recombination breakpoints using the GARD algorithm implemented by the HyPhy software (Kosakovsky P et al., 2006a, b). Then, the processed file was tested for selection using the FEL and IFEL algorithms using the datamonkey server (Kosakovskyi PSL, Frost SD, 2005). Episodic diversifying selection was detected using the MEME algorithm (Murrell B et al., 2012). A p $<0.01$ was considered to be statistically significant for all of the selection tests. The RELAX test to detect relaxed selection on the codon-based phylogenetic framework of alpA from Colombian isolates was performed using the datamonkey server (Kosakovskyi PSL, Frost SD, 2005). 


\section{Results}

A total of 142 sequences were used in this study: 86 were obtained from Colombian patients with different gastric diseases, and 34 sequences were obtained from reference strains from GenBank. The phylogenetic tree of AlpA using only the reference pool showed three clades: one clustering sequences from HspAmerind and HspAsia populations, the second clustering sequences from HpEurope and HspSouthIndia, and the last clustering sequences exclusively from HspWestAfrica. The tree does not reflect a clear separation between HspAmerind and HspAsia nor between HpEurope and HspSouthIndia, which suggests that recombination and gene conversion gave rise to its evolutionary pattern (Figure 1).

The phylogenetic tree including Colombian isolates showed an intricate pattern of clades. Five major clades were detected: 1) Cluster sequences from HspWestAfrica, HspColombia and HpEurope; 2) Cluster sequences from HpEurope, HspSouthIndia and HspColombia; 3) Cluster sequences from HspColombia; 4) Cluster sequences from HspAsia, HspAmerind; and 5) Cluster sequences from HpEurope, HspAsia and HspColombia (Figure 2). The phylogenetic tree of the Colombian isolates showed three major clades, called Col1, Col2 and Col3; a more detailed analysis allowed us to show that the phylogenetic tree had seven subclades, called 1 to 7 , which indicates that an intense evolutionary process is taking place between Colombian isolates with respect to AlpA (Figure 3).

The analysis of the nucleotide diversity (2.5-fold) and the average number of nucleotide differences (3.7-fold) showed them to be higher in the reference pool than in the Colombian isolates. Similarly, the theta estimator showed that the reference pool was significantly more diverse than the Colombian counterparts. The number of haplotypes was 3-fold higher in the Colombian isolates than that observed in the reference pool, but the haplotype diversity was similarly higher in both populations; the total number of haplotypes was 134, with an extreme value for the haplotype diversity. Recombination events were 1.3 -fold higher in the Colombian isolates than in the reference pool (Table 1).

To test the deviations of the neutral model of molecular evolution, the Tajima and Z-test were employed. The Tajima's D test was negative and the Z-test showed significant results for neutrality, indicating that in overall average the synonymous substitution rate was similar the non-synonymous substitution rate $(\mathrm{p}=0.005)$ (Table 2$)$. However, the sliding windows analysis showed that several regions of the alp $A$ has an $\omega$ value above 1, which suggests that episodic positive diversifying selection is also shaping the microevolutionary patterns of this gene in Colombia (Figure 4).

The test of genetic differentiation showed that the Colombian population was well-differentiated from the reference pool, but as expected, the Nm obtained from FsT was 3.63, which indicates a moderate gene flow between the Colombian isolates (Slatkin, 1985, 1987) (Table 3). To assess the isolation between pairs of populations, the Colombian isolates were organized into seven groups based on the histopathological diagnosis, and then, the alpA DNA sequences were compared pairwise with the reference pool clustered according to their phylogeographic classification. Population isolation was found between the Colombian populations and all subpopulations (Table 4). Two gene conversion algorithms were applied to the population to test 
220

221

222

223

224

225

226

227

228

229

230

231

232

233

234

235

236

237

238

239

240

241

242

243

244

245

246

247

248

249

250

251

252

253

254

255

256

257

258

259

260

261

262

263

264

265

whether this process was involved in the evolutionary pattern of alp $A$. Betran's algorithm found 54 conversion tracks between the sequences: $20.5 \%$ for the reference pool and $44.4 \%$ for the Colombian isolates, and the genconv algorithm found 6 tracks of gene conversion between the sequences that belong exclusively to the Colombian isolates (Figure 5).

Functional divergence analysis among the AlpA proteins sequences based on the three major clades found in the phylogenetic tree of the Colombian isolates was performed using Gu's type-I method. The pairwise comparisons between the Col1/Col3 clades showed five sites with a $\theta \mathrm{D}>$ 0.8 (Figure $3 \mathrm{~B}$ ), which indicates that the protein presents sites with different evolutionary rates. The analysis of positive and negative selection covered 540 codon sites of the AlpA protein. A similar number of sites were detected by the FEL and IFEL algorithms. FEL identified that 3.3\% of the sites were under positive selection and $21.4 \%$ evolve under purifying selection. IFEL showed that $2.7 \%$ of the sites evolve under positive selection, with $12.2 \%$ under purifying selection, and finally, MEME showed that $5 \%$ of the adhesin was under episodic diversifying selection (Figure 6). The relax test showed that when the internal branches were compared against the external branches, a significant pattern of natural selection intensification was detected $\left(\mathrm{K}=29.55, p=5.48 \mathrm{e}^{-10}, \mathrm{LR}=38.50\right)$.

\section{Discussion}

In this study, we describe the genetic diversity and microevolution of AlpA in Colombian isolates of $H$. pylori obtained from a high gastric cancer region composed of the cities of Bogotá, Tunja and the surrounding towns. The phylogenetic tree using the reference AlpA sequences revealed that the strains clustered according to their geographic origin; two main clades were observed: the Eastern and the Western clades. Then, within each main clade, there was no separation between the $H$. pylori subpopulations. This geographical segregation has been observed for other virulence factors of $H$. pylori (Cao P et al., 2005; Maeda S et al., 1998; Oleastro M et al., 2009a; Van Doorn LJ et al., 1999) and it is an indicative of the precolonization period. However, when the sequences of Colombian isolates are added to the analysis, the main East and Western clades fade, which indicates that the sequences share information by recombination and diversifying selection induced by the post-colonization period, when the host and bacterial populations mix together in Latin-America, and for the induction of different divergence rates between the paralogous/homologous members of different strains as a consequence of gene conversion (Santoyo G and Romero D, 2005).

The phylogenetic tree of Colombian isolates showed tree dominant clades entitled Col1, Col2 and Col3. The clades Col1 and Col2 have few branching events with a low number of members, but the Col3 clade showed five subclades. There was no association between the disease state and belonging to a specific clade ( $\mathrm{p}=0.245$ Chi-square 2.81), which indicates that the $H$. pylori strains interchange DNA randomly between the Colombian strains. It is a well-established fact that the bacterium is naturally competent (Dorer MS et al., 2013).

The nucleotide diversity and the average number of nucleotide differences was lower in the Colombian alp $A$ alleles that in the reference pool. However, the Colombian isolates showed a higher number of haplotypes and recombination events. The alp $A$ gene from Colombian isolates has shown strong patterns of genetic differentiation, but it maintains the genetic flow that has 
266

267

268

269

270

271

272

273

274

275

276

277

278

279

280

281

282

283

284

285

286

287

288

289

290

291

292

293

294

295

296

297

298

299

300

301

302

303

304

305

306

307

308

309

310

311

produced new allelic variants of alpA in Colombia. The arrival of the HpEurope to LatinAmerica induced the replacement of the native HspAmerind population from urban zones (Yamaoka Y et al., 2002) and perhaps produced a selective bottleneck. After the bottleneck, a subsequent population expansion emerged with the new recombinant $H$. pylori subtypes in Colombia. It has been proposed that recombination among $H$. pylori strains can induce the evolution of different subclones and genotypes (Blaser and Berg, 2001; Blaser and Atherton, 2004).

H. pylori has one of the highest mutation and recombination rates between bacterial species (Suerbaum \& Josenhans, 2007). It has been identified that $H$. pylori has followed unique evolutionary pathways in Latin-America (Muñoz-Ramírez ZY et al., 2017), and the strains have followed rapid adaptive processes in different countries of the region (Gutiérrez-Escobar AJ et al., 2017; Thorell K et al., 2017). Another factor that contributes to the rapid evolution of the Colombian $H$. pylori alpA subtypes was the mestizo host. Perhaps the immune and inflammatory responses of this new host and its genetic heterogeneity represented by the variability and distribution of receptors to which the bacterium can adhere could be a selective factor for the divergence among Colombian H. pylori strains (Dubois A et al., 1999; Oleastro M et al., 2009b). In fact, the host imposes a selective pressure that induces variation within the bacterium (Thompson et al., 2004).

Gene conversion was a major process that gave rise to the allelic variation of alpA in Colombia, and the paralogous interchange of the DNA fragments close to the 3' region of the gene was considerable higher between the Colombian isolates than in the reference pool. In total, 60 recombination events were detected by the two applied algorithms, and despite the total number of sequences used in the analysis, only Colombian alp $A$ alleles showed statistical signals of this type of recombination. Gene conversion has been identified previously in other $H$. pylori adhesins, for example, homB and homA (Dorer MS et al., 2013; Dubois A et al., 1999; Oleastro M et al., 2009a; Oleastro M et al., 2009b; Santoyo G Romero D, 2005; Yamaoka Y et al., 2002), $s a b B$ and omp27 (Talarico S et al., 2012) and babA (Hennig EE et al., 2006), and a LatinAmerican type of $b a b A$ has also been recently reported (Thorell $\mathrm{K}$ et al., 2016).

The interplay between positive selection and recombination has been detected in bacterial genomes (Lefebure \& Stanhope, 2007; Joseph et al., 2011; Orsi et al., 2008). The analysis of functional divergence analysis of the protein AlpA based on the comparison of the tree clades Col1, Col2 and Col3 showed that the protein residues 23V, 65V, 190Q, 219A and 446G have a significant evolutionary site variation rate with a $\theta \mathrm{D}$ value of 0.8 , which confers functional differences between the members of the clades Coll/Col3. In Colombia, positive, episodic diversifying, purifying selection and recombination of alp $A$ has given rise to the presence of rare alleles and new haplotypes in the emerging population, which have been maintained at the protein level by natural selection.

The action of natural selection might purge diversified genes from the population by strong purifying selection (Lynch M, Conery JS, 2000) or lead to evolutionary novelties by positive selection (Wertheim JO et al., 2015). When the internal branches of the phylogenetic tree of Colombian alp $A$ alleles were compared against the external ones, we determined that there was a significant natural selection intensification operating in the $\operatorname{alp} A$ alleles $\left(\mathrm{K}=29.55, p=5.48 \mathrm{e}^{-10}\right.$, 
$\mathrm{LR}=38.50$ ), which means that positive selection is leading the microevolution at the high gastric cancer risk region of Colombia.

The Mestizo populations in the mountain zones in Colombia have an admixture of European and Amerind ancestries in a similar fashion that the bacterium mirroring the colonization process (Kodaman $\mathrm{N}$ et al., 2014). When the Colombian H. pylori strains and human host ancestries are compared, a difference in the intensification of the disease aggressiveness is observed. Deleterious duplicated alleles of alpA were purged out of the population, but those strains with fixed alp $A$ alleles under positive selection give advantages to the new types of strains in this region.

H. pylori is a bacterium that can display very fast local adaptive processes via mutation and recombination (Cao Q et al., 2015; Furuta Y et al., 2015). The phylogenetics, population genetics and protein evolutionary analysis suggest that alp $A$ in Colombia has functionally divergent variants that are the result of gene conversion in a staggeringly short period of time. AlpA proteins from the Colombian population show evidence of functional divergence, positive selection and episodic positive selection at specific sites. It is possible that the polymorphism of this adhesin in Colombia reflects the phylogeography and historical generation of Mestizos in Latin-America because $H$. pylori is a reliable biological marker of human migratory events (Templeton AR, 2007).

\section{Conclusion}

The molecular evolution of virulence factors of $H$. pylori is currently gaining attention in the scientific community due to the new genomics and evolutionary findings around the world. Currently, Latin-American countries have emerged as evolutionary laboratories for H. pylori. To our knowledge, this study is the first study that presents statistically supported evidence that alp $A$ alleles from a high gastric cancer risk area from Colombia owe their variation patterns to gene conversion and purifying selection. In addition, a fast process of gene diversification followed by positive and relaxed selection has shaped three protein variants for the AlpA adhesin in Colombia.

\section{Acknowledgements}

Thank to the anonymous reviewers for the constructive comments and guidelines.

\section{References}

Achtman M, Azuma T, Berg DE, Ito Y, Morelli G, Pan ZJ, Suerbaum S, Thompson SA, van der Ende A and van Doorn LJ. 1999. Recombination and clonal groupings within Helicobacter pylori from different geographical regions. Mol Microbiol 32(3): 459-70.

Alm RA, Bina J, Andrews, BM, Doig P, Hancock RE and Trust TJ. 2000. Comparative genomics of Helicobacter pylori: Analysis of the outer membrane protein families. Infect Immun 68(7): 4155-68. 
358

359

360

361

362

363

364

365

366

367

368

369

370

371

372

373

374

375

376

377

378

379

380

381

382

383

384

385

386

387

388

389

390

391

392

393

394

395

396

397

398

399

400

401

402

403
Bertuccio P, Chatenoud L, Levi F, Praud D, Ferlay J, Negri E, Malvezzi M, La Vecchia C. 2009. Recent patterns in gastric cancer: a global overview, Int. J. Cancer 125 (3): 666-673.

Betran E, Rozas J, Navarro A and Barbadilla A. 1997. The estimation of the number and the length distribution of gene conversion tracts from population DNA sequence data. Genetics 146(1): 89-99.

Bianchine PJ and Russo TA. 1992. The role of epidemic infectious diseases in the discovery of America. Allergy Proc 13(5): 225-32.

Blaser MJ \& Atherton JC. 2004. Helicobacter pylori persistence: biology and disease. J. Clin.Invest. 113, 321-333.

Blaser MJ \& Berg DE. 2001. Helicobacter pylori genetic diversity and risk of human disease. J. Clin. Invest. 107, 767-773.

Cao P, Lee KJ, Blaser MJ and Cover TL. 2005. Analysis of hopQ alleles in East Asian and Western strains of Helicobacter pylori. FEMS Microbiol Lett 251(1): 37-43.

Cao Q, Didelot X, Wu Z, Li Z, He L, Li Y, Ni M, You Y, Lin X, Li Z, Gong Y, Zheng M, Zhang M, Liu J, Wang W, Bo X, Falush D, Wang S and Zhang J. 2015. Progressive genomic convergence of two Helicobacter pylori strains during mixed infection of a patient with chronic gastritis. Gut 64(4): 554-61.

Correa P \& Piazuelo B. (2010). Gastric cancer: The colombian enigma. Revista Colombiana de Gastroenterologia, 25(4), 334-337.

Devi SM, Ahmed I, Francalacci P, Hussain MA, Akhter Y, Alvi A, Sechi LA, Mégraud F, Ahmed N. 2007. Ancestral European roots of Helicobacter pylori in India. BMC Genomics 8: 184.

Dorer MS, Cohen IE, Sessler TH, Fero J and Salama NR. 2013. Natural competence promotes Helicobacter pylori chronic infection. Infect Immun 81(1): 209-15.

Dubois, A, Berg DE, Incecik ET, Fiala N, Heman-Ackah LM, Del Valle J, Yang M, Wirth HP, Perez-Perez GI and Blaser MJ. 1999. Host specificity of Helicobacter pylori strains and host responses in experimentally challenged non-human primates. Gastroenterology 116(1): 90-6.

Edgar RC. 2004. MUSCLE: multiple sequence alignment with high accuracy and high throughput. Nucleic Acids Res 32(5): 1792-7.

Falush D, Wirth T, Linz B, Pritchard JK, Stephens M, Kidd M, Blaser MJ, Graham DY, Vacher S, Perez-Perez GI, Yamaoka Y, Mégraud F, Otto K, Reichard U, Katzowitsch E, Wang X, Achtman M and Suerbaum S. 2003. Traces of human migrations in Helicobacter pylori populations. Science 299(5612): 1582-5. 
404 Ferlay J, Soerjomataram I, Ervik M, Dikshit R, Eser S, Mathers C, Rebelo M, Parkin DM, 405 Forman D, Bray, F. 2013, GLOBOCAN 2012 v1.0, Cancer Incidence and Mortality Worldwide: 406 IARC Cancer Base No.11, International Agency for Research on Cancer, Available from: URL: 407 http://globocan.iarc.fr.

408

409

410

411

412

413

414

415

416

417

418

419

420

421

422

423

424

425

426

427

428

429

430

431

432

433

434

435

436

437

438

439

440

441

442

443

444

445

446

447

448

449

Forman D \& Sierra MS. 2014. The current and projected global burden of gastric cancer. Helicobacter pylori Eradication as a Strategy for Preventing Gastric Cancer. IARC Helicobacter pylori Working Group. Lyon, France: International Agency for Research on Cancer (IARC Working Group Reports, No. 8). 5, 15.

Furuta Y, Konno M, Osaki T, Yonezawa H, Ishige T, Imai M, Shiwa Y, Shibata-Hatta M, Kanesaki Y, Yoshikawa H, Kamiya S and Kobayashi I. 2015. Microevolution of virulencerelated genes in Helicobacter pylori familial infection. PLoS One 10(5): e0127197.

Gu X, Zou Y, Su Z, Huang W, Zhou Z, Arendsee Z and Zeng Y. 2013. An update of DIVERGE software for functional divergence analysis of protein family. Mol Biol Evol 30(7): 1713-9.

$\mathrm{Gu}$ X. Statistical methods for testing functional divergence after gene duplication.1999. Mol Biol Evol 16(12): 1664-74.

Gutiérrez-Escobar AJ, Trujillo E, Orlando E. Acevedo S, Bravo MM. 2017. Phylogenomics of Colombian Helicobacter pylori isolates. Gut Pathog, 9:52-61.

Hartl DL \& Clark GC. 1997. Principles of Population Genetics. Sinauer Associates,Sunderland.

Helicobacter and Cancer Collaborative Group. Gastric cancer and Helicobacter pylori: A combined analysis of 12 case control studies nested within prospective cohorts. Gut. 2001;49:347-53.

Hennig EE, Allen JM and Cover TL. 2006. Multiple chromosomal loci for the babA gene in Helicobacter pylori. Infect Immun 74(5): 3046-3051.

Jemal A, Center MM, DeSantis C, Ward EM. Global patterns of cancer incidence and mortality rates and trends, Cancer Epidemiol. Biomarker Prev. 19 (8) (2010) 1893-1907.

Joseph SJ, Didelot X. Gandhi K, Dean D, Read TD. 2011. Interplay of recombination and selection in the genomes of Chlamydia trachomatis, Biol. Direct., 6, 28.

Khalifa MM, Sharaf RR and Aziz RK. 2010. Helicobacter pylori: a poor man's gut pathogen? Gut Pathog 2: 2.

Kodaman N, Pazos A, Schneider BG, Piazuelo MB, Mera R, Sobota RS, Sicinschi LA, Shaffer CL, Romero-Gallo J, de Sablet T, Harder RH, Bravo LE, Peek RM Jr, Wilson KT, Cover TL, Williams SM and Correa P. 2014. Human and Helicobacter pylori coevolution shapes the risk of gastric disease. Proc Natl Acad Sci U S A 111(4): 1455-1460. 
450 Kosakovsky Pond SL and Frost SD. 2005. Not so different after all: a comparison of methods for

451

452

453

454

455

456

457

458

459

460

461

462

463

464

465

466

467

468

469

470

471

472

473

474

475

476

477

478

479

480

481

482

483

484

485

486

487

488

489

490

491

492

493

494 detecting amino acid sites under selection. Mol Biol Evol 22(5): 1208-22.

Kosakovsky Pond SL, Posada D, Gravenor MB, Woelk CH, Frost SD. 2006. GARD: a genetic algorithm for recombination detection. Bioinformatics 22: 3096-3098.

Kosakovsky Pond SL, Posada D, Gravenor MB, Woelk CH, Frost SD. 2006. Automated phylogenetic detection of recombination using a genetic algorithm. Mol Biol Evol 23 (10):1891901.

Kumar S Stecher G, Tamura K . 2016. MEGA7: molecular evolutionary genetics analysis version 7.0 for bigger data sets. Mol Biol Evol. 33(7):1870-1874.

Lefebure T. \& Stanhope MJ. 2007. Evolution of the core and pangenome of Streptococcus: positive selection, recombination, and genome composition, Genome Biol., 8, R71.

Librado P and Rozas J. 2009. DnaSP v5: a software for comprehensive analysis of DNA polymorphism data. Bioinformatics 25(11): 1451-2.

Linz B, Balloux F, Moodley Y, Manica A, Liu H, Roumagnac P, Falush D, Stamer C, Prugnolle F, van der Merwe SW, Yamaoka Y, Graham DY, Perez-Trallero E, Wadstrom T, Suerbaum S and Achtman M. 2007. An African origin for the intimate association between humans and Helicobacter pylori. Nature 445(7130): 915-918.

Lu H, Wu JY, Beswick EJ, Ohno T, Odenbreit S, Haas R, Reyes VE, Kita M, Graham DY and Yamaoka Y. 2007. Functional and intracellular signalling differences associated with the Helicobacter pylori AlpAB adhesin from Western and East Asian strains. J Biol Chem 282(9): $6242-54$.

Lynch M and Conery JS. 2000. The evolutionary fate and consequences of duplicate genes. Science 290(5494): 1151-5.

Maeda S, Ogura K, Yoshida H, Kanai F, Ikenoue T, Kato N, Shiratori Y and Omata M. 1998. Major virulence factors, VacA and CagA, are commonly positive in Helicobacter pylori isolates in Japan. Gut 42(3): 338-43.

Mahdavi J, Sondén B, Hurtig M, Olfat FO, Forsberg L, Roche N, Angstrom J, Larsson T, Teneberg S, Karlsson KA, Altraja S, Wadström T, Kersulyte D, Berg DE, Dubois A, Petersson C, Magnusson KE, Norberg T, Lindh F, Lundskog BB, Arnqvist A, Hammarström L and Borén T. 2002. Helicobacter pylori SabA adhesin in persistent infection and chronic inflammation. Science 297(5581): 573-8.

Matsuo Y, Kido Y, Yamaoka Y. 2017. Helicobacter pylori outer membrane protein-related pathogenesis. Toxins 9(3): 101. 
498

499

500

501

502

503

504

505

506

507

508

509

510

511

512

513

514

515

516

517

518

519

520

521

522

523

524

525

526

527

528

529

530

531

532

533

534

535

536

537

538

539

540

Matta AJ, Pazos AJ, Bustamante-Rengifo JA, Bravo LE. Genomic variability of Helicobacter pylori isolates of gastric regions from two Colombian populations. World J Gastroenterol. 2017 Feb 7;23(5):800-809.

Moodley Y, Linz B, Yamaoka Y, Windsor HM, Breurec S, Wu JY, Maady A, Bernhöft S, Thiberge JM, Phuanukoonnon S, Jobb G, Siba P, Graham DY, Marshall BJ and Achtman M. 2009. The peopling of the Pacific from a bacterial perspective. Science 323(5913): 527-30.

Muñoz-Ramírez ZY, Mendez-Tenorio A, Kato I, Bravo MM, Rizzato C, Thorell K, Torres R, Aviles-Jimenez F, Camorlinga M, Canzian F and Torres J. 2017. Whole genome sequence and phylogenetic analysis show Helicobacter pylori strains from Latin America have followed a unique evolution pathway. Front Cell Infect Microbiol 7: 50.

Murrell B, Wertheim JO, Moola S, Weighill T, Scheffler K, Kosakovsky and Pond SL. 2012. Detecting individual sites subject to episodic diversifying selection. PLoS Genet 8(7): e1002764.

Nomura AMY, Perez Perez GI, Lee J, Stemmermann G, Blaser MJ. 2002. Relation between Helicobacter pylori cagA status and risk of peptic ulcer disease. Am J Epidemiol 155(11): 10549.

Odenbreit S, Swoboda K, Barwig I, Ruhl S, Borén T, Koletzko S and Haas R. 2009. Outer membrane protein expression profile in Helicobacter pylori clinical isolates. Infect Immun 77(9): 3782-90.

Odenbreit S, Till M, Hofreuter D, Faller G and Haas R. 1999. Genetic and functional characterization of the alpAB gene locus essential for the adhesion of Helicobacter pylori to human gastric tissue. Mol Microbiol 31(5): 1537-48.

Oleastro MR, Cordeiro R, Ménard A, Yamaoka Y, Queiroz D, Mégraud F anb Monteiro L. 2009a. Allelic diversity and phylogeny of homB, a novel co-virulence marker of Helicobacter pylori. BMC Microbiol 9: 248.

Oleastro MR, Cordeiro Y, Yamaoka D, Queiroz F, Mégraud L, Monteiro and Ménard A. 2009b. Disease association with two Helicobacter pylori duplicate outer membrane protein genes, homB and homA. Gut Pathog 1(1): 12.

Orsi RH, Sun Q, Wiedmann M. 2008. Genome-wide analyses reveal lineage specific contributions of positive selection and recombination to the evolution of Listeria monocytogenes, BMC Evol. Biol., 8, 233.

Parrish CR, Holmes EC, Morens DM, Park EC, Burke DS, Calisher CH, Laughlin CA, Saif LJ and Daszak P. 2008. Cross-species virus transmission and the emergence of new epidemic diseases. Microbiol Mol Biol Rev 72(3): 457-470.

Parsonnet J, Friedman GD, Orentreich N and Vogelman H. 1997. Risk for gastric cancer in people with CagA positive or CagA negative Helicobacter pylori infection. Gut 40(3): 297-301. 
541 Pawlowski M, Gajda MJ, Matlak R and Bujnicki JM. 2008. MetaMQAP: a meta-server for the 542 quality assessment of protein models. BMC Bioinformatics 9: 403.

543

544

545

546

547

548

549

550

551

552

553

554

555

556

557

558

559

560

561

562

563

564

565

566

567

568

569

570

571

572

573

574

575

576

577

578

579

580

581

582

583

584

Perez-Perez GI, Rothenbacher D and Brenner H. 2004. Epidemiology of Helicobacter pylori infection. Helicobacter 9 (Suppl. 1): 1-6.

Saitou N and Nei M. 1987. The neighbour-joining method: a new method for reconstructing phylogenetic trees. Mol Biol Evol 4(4): 406-25.

Santoyo G and Romero D. 2005. Gene conversion and concerted evolution in bacterial genomes. FEMS Microbiol Lett 29(2): 169-83.

Sawyer S. 1989. Statistical tests for detecting gene conversion. Mol Biol Evol 6(5): 526-38.

Slatkin M. 1985. Gene flow in natural populations. Ann. Rev. Ecol. Syst. 16:393-430.

Slatkin M. 1987. Gene flow and the geographic structure of natural populations. Science 236:787-792.

Suerbaum S. and Josenhans C. 2007. Helicobacter pylori evolution and phenotypic diversification in a changing host, Nat. Rev. Microbiol., 5, 441-52.

Sun ZL, Bi YW, Bai CM, Gao DD, Li ZH, Dai ZX, Li JF and Xu WM. 2010. Expression of Helicobacter pylori alpA gene in Lactococcus lactis and its immunogenicity analysis. Xi Bao Yu Fen Zi Mian Yi Xue Za Zhi 26(3): 203-6.

Tajima F. 1989. Statistical method for testing the neutral mutation hypothesis by DNA polymorphism. Genetics 123(3): 585-95.

Talarico S, Whitefield SE, Fero J, Haas R and Salama NR. 2012. Regulation of Helicobacter pylori adherence by gene conversion. Mol Microbiol 84(6): 1050-61.

Templeton AR. 2007. Shared history of humans and gut bacteria: evolutionary togetherness: coupled evolution of humans and a pathogen. Heredity 98(6): 337-8.

Thompson LJ, Danon SJ, Wilson JE, O'Rourke JL, Salama NR, Falkow S, Mitchell H, Lee A. 2004. Chronic Helicobacter pylori infection with Sydney strain 1 and a newly identified mouseadapted strain (Sydneystrain2000) in C57BL/6 and BALB/c mice. Infect.Immun. 72, 4668-4679.

Thorell K, Hosseini S, Palacios Gonzáles RV, Chaotham C, Graham DY, Paszat L, Rabeneck L, Lundin SB, Nookaew I and Sjöling Å. 2016. Identification of a Latin American-specific BabA adhesin variant through whole genome sequencing of Helicobacter pylori patient isolates from Nicaragua. BMC Evol Biol 16: 53. 
585 Thorell K, Yahara K, Berthenet E, Lawson DJ, Mikhail J, Kato I, Mendez A, Rizzato C, Bravo

586 MM, Suzuki R, Yamaoka Y, Torres J, Sheppard SK and Falush D. 2017. Rapid evolution of

587 distinct Helicobacter pylori subpopulations in the Americas. PLoS Genet 13(2): e1006546.

588

589

590

Torres J, Correa P, Ferreccio C, Hernandez-Suarez G, Herrero R, Cavazza-Porro M, Dominguez

591

592

593

594

595

596

597

598

599

600

601

602

603

604

605

606

607

608

609

610

611

612 R, Morgan D. 2013. Gastric cancer incidence and mortality is associated with altitude in the mountainous regions of Pacific Latin America. Cancer Causes Control 24 (2): 249-256.

Van Doorn LJ, Figueiredo C, Mégraud F, Pena S, Midolo P, Queiroz DM, Carneiro F, Vanderborght B, Pegado MD, Sanna R, De Boer W, Schneeberger PM, Correa P, Ng EK, Atherton J, Blaser MJ and Quint WG. 1999. Geographic distribution of vacA allelic types of Helicobacter pylori. Gastroenterology 116(4):823-30.

Wertheim JO, Murrell B, Smith MD, Kosakovsky-Pond SL and Scheffler K. 2015. RELAX: detecting relaxed selection in a phylogenetic framework. Mol Biol Evol 32(3): 820-32.

Xue J, Bai Y, Chen Y, Wang JD, Zhang ZS, Zhang YL and Zhou DY. 2005. Expression of Helicobacter pylori AlpA protein and its immunogenicity. World J Gastroenterol 11(15): 22602263.

Yakirevich E and Resnick MB. 2013. Pathology of gastric cancer and its precursor lesions. Gastroenterol Clin North Am 42(2): 261-84.

Yamaoka Y, Kikuchi S, El-Zimaity HMT, Gutierrez O, Osato MS, Graham DY. 2002. Importance of Helicobacter pylori oipA in clinical presentation, gastric inflammation, and mucosal interleukin 8 production. Gastroenterology 123(2): 414-24.

Yamaoka Y, Orito E, Mizokami M, Gutierrez O, Saitou N, Kodama T, Osato MS, Kim JG, Ramirez FC, Mahachai V and Graham DY. 2002. Helicobacter pylori in North and South America before Columbus. FEBS Lett 517(1-3): 180-4.

615

616

617

Yamaoka Y. 2009. Helicobacter pylori typing as a tool for tracking human migration. Clin Microbiol Infect 15(9): 829-34.

618

619 Yang J, Yan R, Roy A, Xu D, Poisson J and Zhang Y. 2015. The I-TASSER Suite: Protein 620 structure and function prediction. Nat Methods 12(1): 7-8. 


\section{Figure 1}

Phylogenetic tree of AlpA from reference strains of Helicobacter pylori.

The evolutionary history was inferred using the Neighbor-Joining method. The optimal tree with the sum of branch length $=0.84663616$ is shown. The percentage of replicate trees in which the associated taxa clustered together in the bootstrap test (1000 replicates); significant consensus tree branches are showed. The evolutionary distances were computed using the JTT matrix-based method and are in the units of the number of amino acid substitutions per site. The rate variation among sites was modeled with a gamma distribution (shape parameter $=2$ ). The analysis involved 34 amino acid sequences. A) Clade East. B) Clade Western. All positions containing gaps and missing data were eliminated. There were a total of 451 positions in the final dataset.

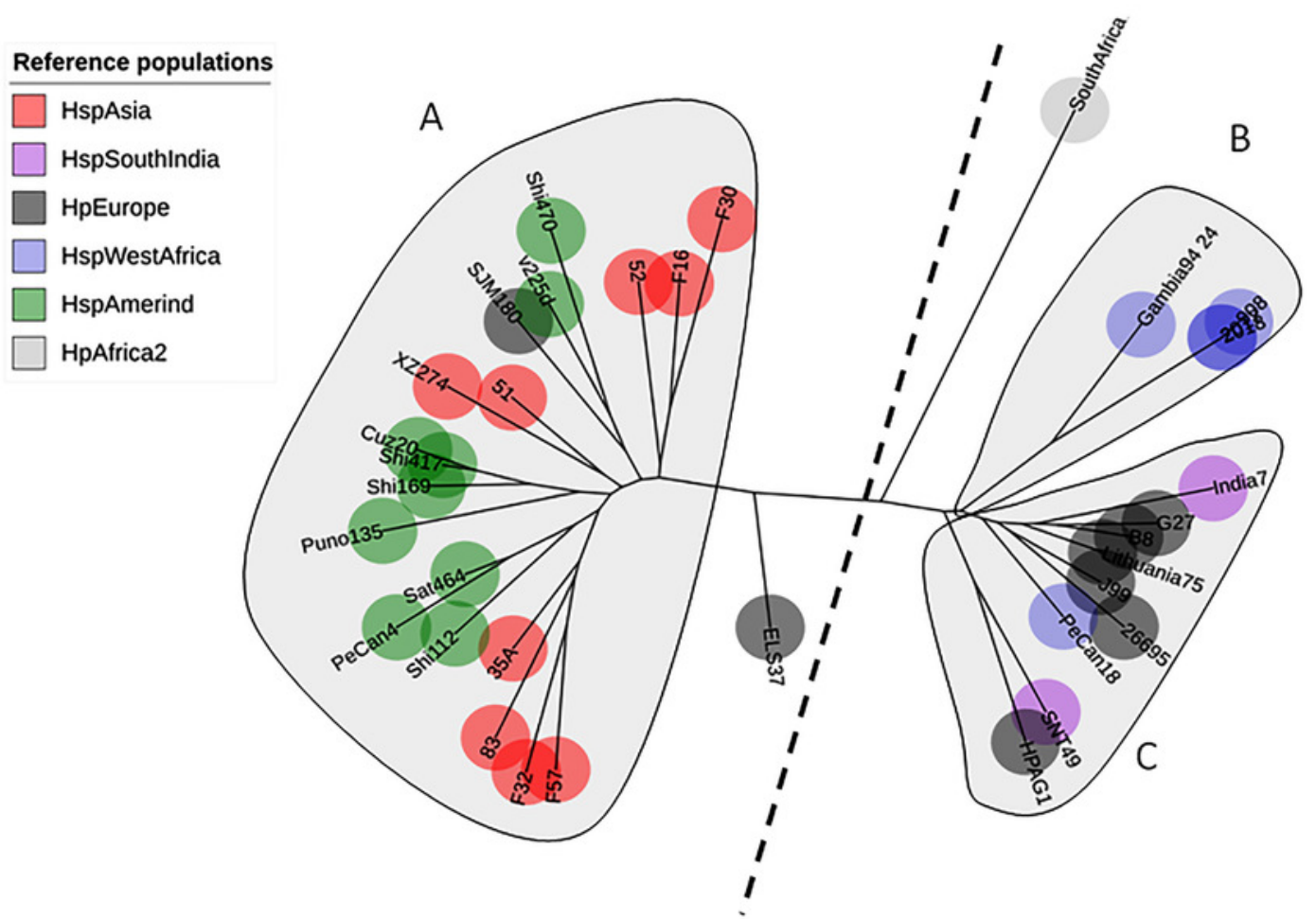




\section{Figure 2}

Phylogenetic tree of AlpA proteins of Helicobacter pylori.

The evolutionary history was inferred using the Neighbor-Joining method. The optimal tree with the sum of branch length $=0.84663616$ is shown. The percentage of replicate trees in which the associated taxa clustered together in the bootstrap test (1000 replicates); significant consensus tree branches are showed. The evolutionary distances were computed using the JTT matrix-based method and are in the units of the number of amino acid substitutions per site. The rate variation among sites was modeled with a gamma distribution (shape parameter $=2$ ). The analysis involved 142 amino acid sequences. All positions containing gaps and missing data were eliminated. There were a total of 451 positions in the final dataset. Five major clades were detected: 1) Cluster sequences from HspWestAfrica, HspColombia and HpEurope; 2) Cluster sequences from HpEurope, HspSouthIndia and HspColombia; 3) Cluster sequences from HspColombia; 4) Cluster sequences from HpAsia, HspAmerind and HspColombia; and 5) Cluster sequences from HpEurope, HpAsia and HspColombia. 

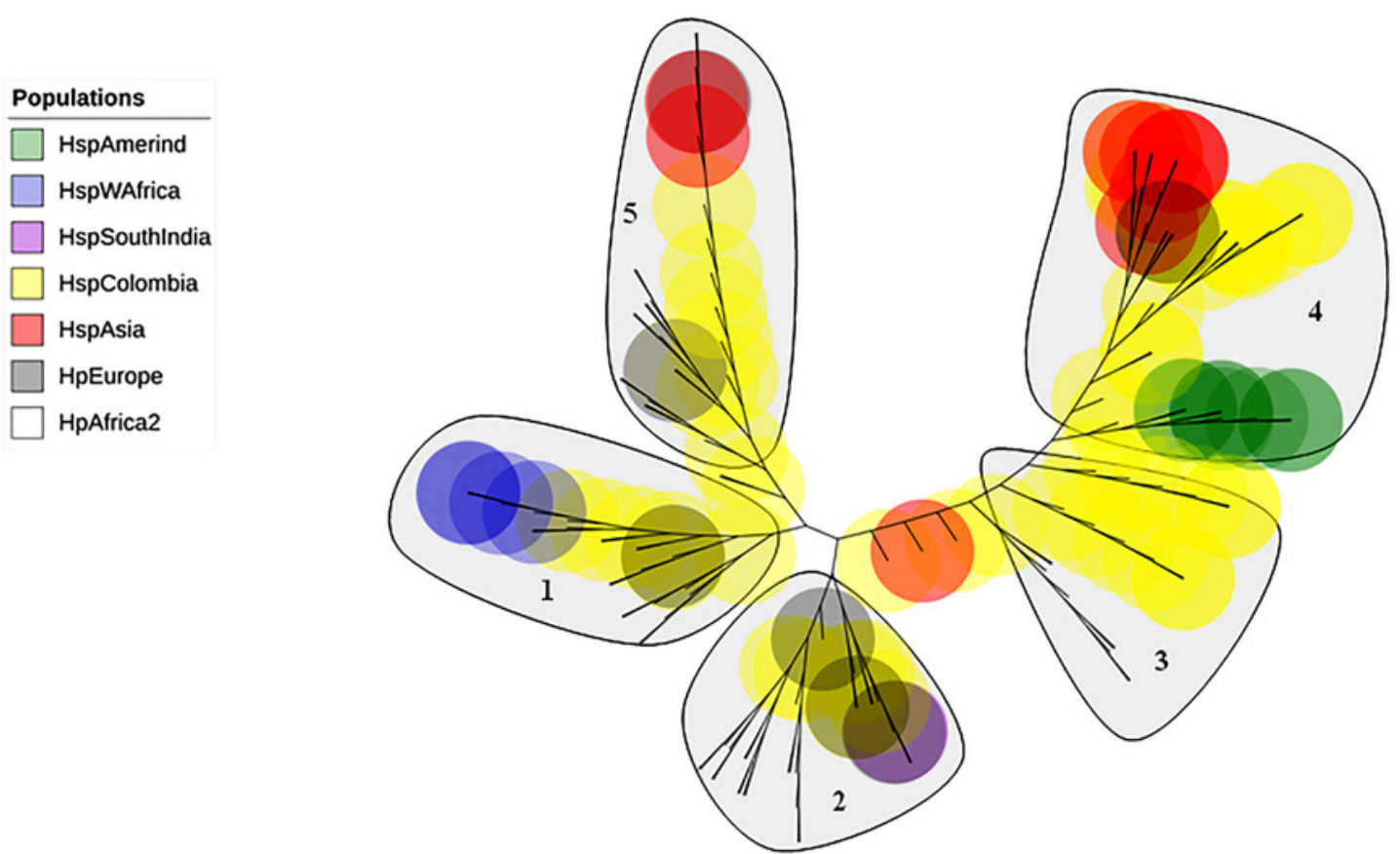


\section{Figure 3}

Phylogenetic tree of AlpA proteins from Colombian isolates of Helicobacter pylori.

A. The evolutionary history was inferred using the Neighbor-joining method. The optimal tree with the sum of branch length $=0.84663616$ is shown. The percentage of replicate trees in which the associated taxa clustered together in the bootstrap test (1000 replicates); significant consensus tree branches are showed. The evolutionary distances were computed using the JTT matrix-based method and are in the units of the number of amino acid substitutions per site. The rate variation among sites was modeled with a gamma distribution (shape parameter $=2$ ). The analysis involved 108 amino acid sequences. All positions containing gaps and missing data were eliminated. There were a total of 451 positions in the final dataset. Three major clades were detected: 1) Col1; 2) Col2 and 3) Col3. B. Comparison all-vs-all between Colombian phylogenetic tree clusters reveals that Col1/Col3 clades have five sites with a $\theta D>0.8$ indicating a strong signal of functional divergence.

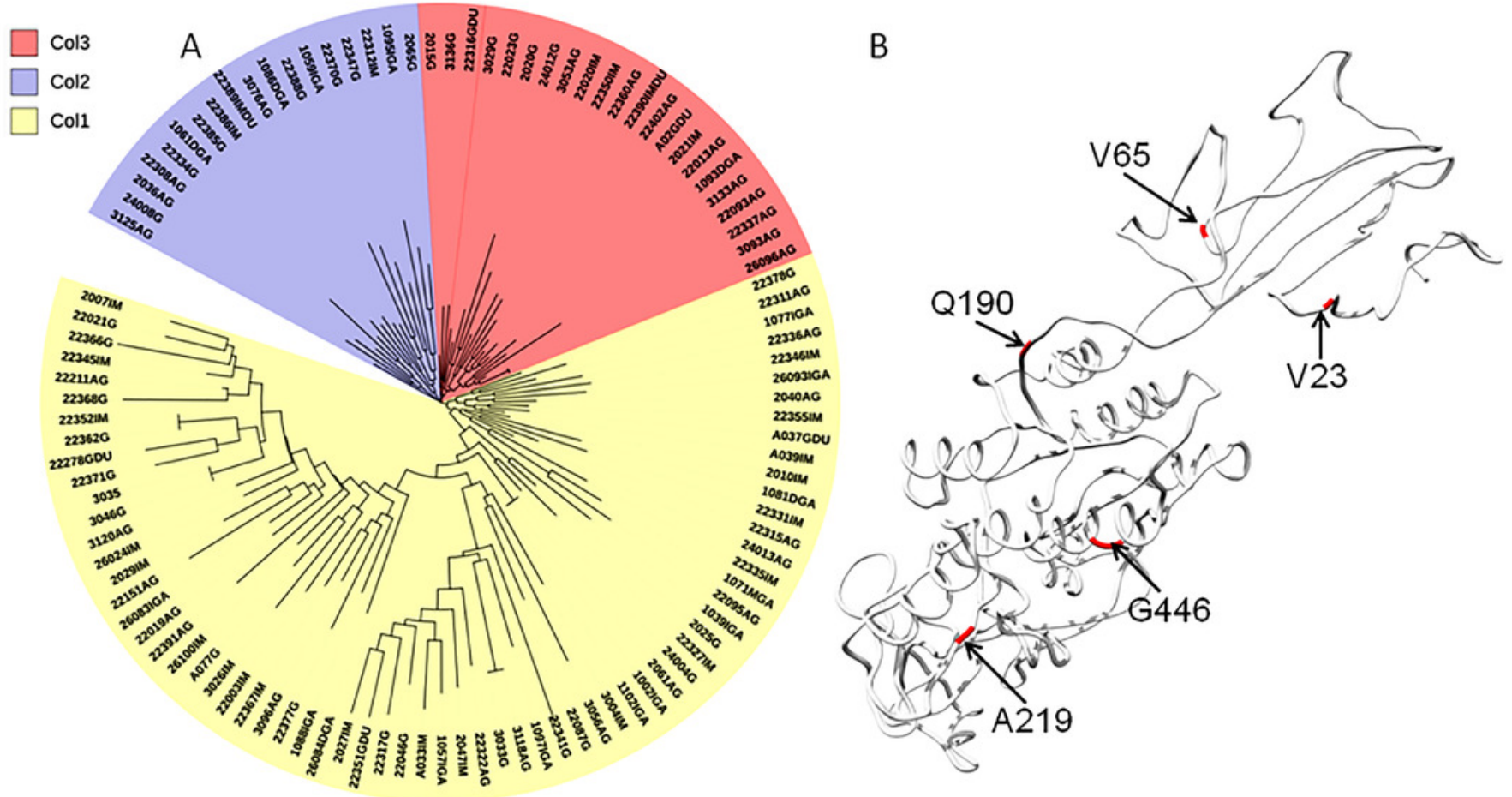


Figure 4

Sliding windows analysis of $\omega$ rate.

Evolutionary rate $\omega=\mathrm{dN} / \mathrm{dS}$ throughout the gene to identify specific regions under natural selection. The analysis involved 142 DNA sequences, 108 from Colombian isolates and 34 as phylogeographic references. The analysis was performed using DnaSP $\vee 5.10$.

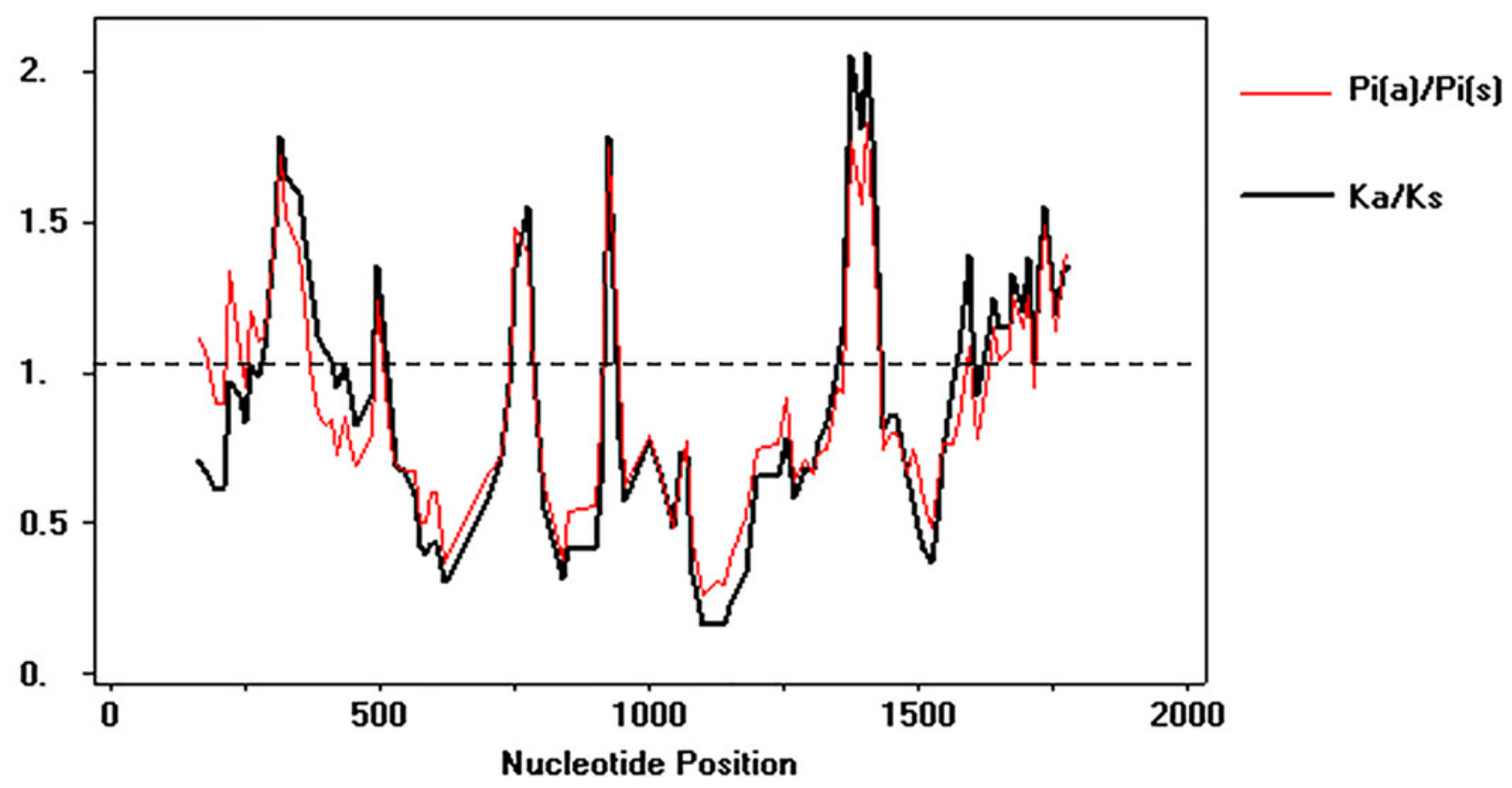




\section{Figure 5}

Gene conversion tracks identified by the GENECONV method.

Each bar is a representation of a gene. The colored rectangles represent the gene fragments or minor parents involved in a gene conversion event. The tracks were located predominantly at the $5^{\prime}$ region of the gene; however some events were also detected at $3^{\prime}$ region. 
$22355 \mathrm{~lm}$

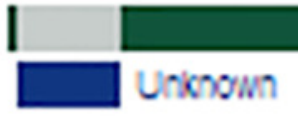

$$
p=0.00003247
$$

$$
p=0.0004783
$$

\section{$22327 \mathrm{M}$}

$p=0.007612$

$$
p=0.01365
$$

$$
p=0.02181
$$

\section{$22380 \mathrm{AG}$}

\section{A037GDU}

$$
\mathrm{p}=0.02478
$$




\section{Figure 6}

Protein model of AlpA showing the positive episodic diversified selected sites after the SBP correction thereby recombination.

A) Positive selected sites were obtained from the tests: FEL (18 sites) and IFEL (14 sites) showed only common sites at the protein structure in red. B) Episodic diversifying selective sites were obtained using the test MEME (27 sites) in black, and both analyses were performed using the datamonkey server from the codon alignment file. Only sites with a $p<$ 0.01 are shown.

A

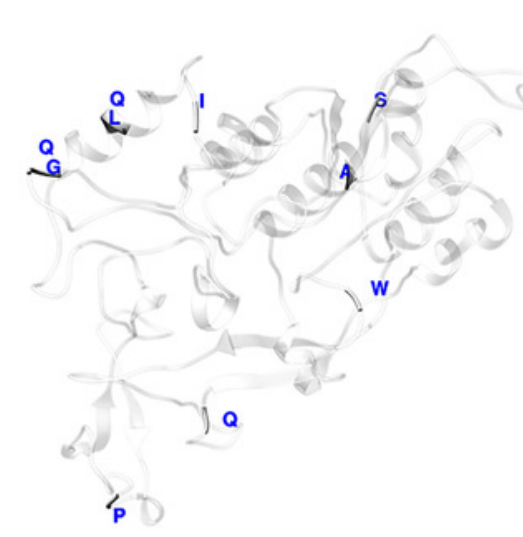

B

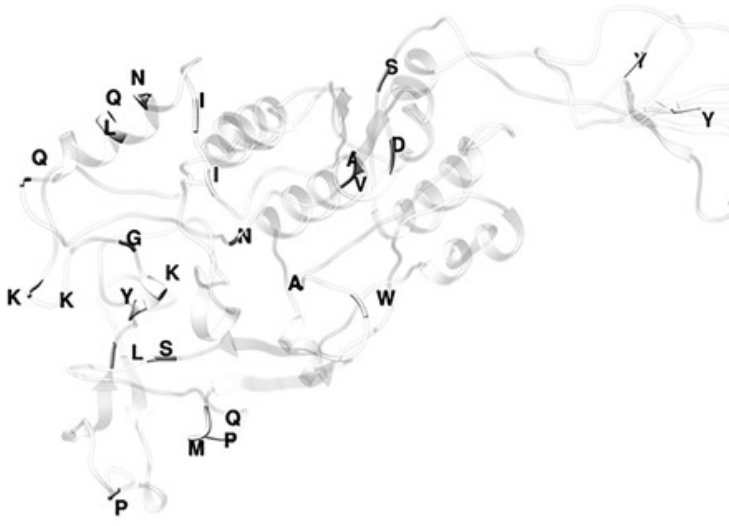




\section{Table $\mathbf{1}$ (on next page)}

Genetic diversity statistics for alpA.

The genetic diversity statistics were calculated by using thirty-four sequences from reference pool and 108 sequences from Colombian isolates of Helicobacter pylori. The estimators were presented in three groups: references, Colombian and both together. n: number of isolates, sites: total number of sites analyzed (excluding gaps), Ss: number of segregant sites, k: average number of nucleotide differences by sequence pairs, $\mathrm{H}$ : number of haplotypes, $\mathrm{Hd}$ : Haplotype diversity, $\theta \mathrm{w}$ : Watterson estimator, $\pi$ : nucleotide diversity per site and RM: recombination events. 
Single Nucleotide Polymorphism

\begin{tabular}{|c|c|c|c|c|c|c|c|c|}
\hline $\mathbf{n}$ & Sites & Ss & $\mathbf{k}$ & $\mathbf{H}$ & Hd & $\theta \mathbf{w}$ & $\pi$ & Rm \\
\hline \multicolumn{9}{|l|}{ Reference } \\
\hline 34 & 1324 & 774 & 200.868 & 33 & 0.998 & 0.143 & 0.152 & 70 \\
\hline \multicolumn{9}{|l|}{ Colombian } \\
\hline 108 & 1384 & 306 & 53.335 & 102 & 0.998 & 0.042 & 0.039 & 91 \\
\hline \multicolumn{9}{|c|}{ Colombian and references } \\
\hline 142 & 1223 & 754 & 81.411 & 134 & 0.999 & 0.112 & 0.067 & 98 \\
\hline
\end{tabular}

2 


\section{Table 2 (on next page)}

Deviations of the neutral model of molecular evolution.

The analysis involved 142 nucleotide sequences. Tajima test: Codon positions included were 1st. All positions containing gaps and missing data were eliminated. There were a total of 359 positions in the final dataset, $\mathrm{m}=$ number of sequences, $\mathrm{n}=$ total number of sites, $\mathrm{S}=$ Number of segregating sites, $\mathrm{ps}=\mathrm{S} / \mathrm{n}, \Theta=\mathrm{ps} / \mathrm{a} 1, \pi=$ nucleotide diversity, and $\mathrm{D}$ is the Tajima statistic. Z-Test: Codon-based test of neutrality for analysis averaging over all sequence pairs. The probability of rejecting the null hypothesis of strict-neutrality $(d N=d S)$ is shown. Values of $\mathrm{P}$ less than 0.05 are considered significant at the $5 \%$ level and are highlighted $\left(* p<0.05,{ }^{* *} p<0.01,{ }^{* * *} p<0.001\right.$; ns: not significative). The test statistics: $d N$ - dS, neutrality; $d N>d S$ positive selection; $d N<d S$ purifying selection are shown. $d S$ and $d N$ are the numbers of synonymous and nonsynonymous substitutions per site, respectively. The variance of the difference was computed using the bootstrap method (1000 replicates). Analyses were conducted using the Nei-Gojobori method. All positions containing gaps and missing data were eliminated. 
Table 2 Deviations of the neutral model of molecular evolution

\section{Tajima test}

\begin{tabular}{|c|c|c|c|c|c|c|c|c|}
\hline & & & & & & \\
\hline$m$ & $S$ & $p_{s}$ & $\boldsymbol{\Theta}$ & $\pi$ & D & $\mathbf{d N}=\mathbf{d S}$ & $d N>d S$ & $\mathrm{dN}<\mathrm{dS}$ \\
\hline 142 & 217 & 0.604 & 0.109 & 0.059 & -1.476 & $-2.878 * * *$ & $-0.213 \mathrm{~ns}$ & $0.214 \mathrm{~ns}$ \\
\hline
\end{tabular}

The analysis involved 142 nucleotide sequences. Tajima test: Codon positions included were 1st. All positions containing gaps and missing data were eliminated. There were a total of 359 positions in the final dataset, $m=$ number of sequences, $n=$ total number of sites, $S=$ Number of segregating sites, $p_{\mathrm{s}}=S / n, \Theta=p_{\mathrm{s}} / \mathrm{a}_{1}, \pi=$ nucleotide diversity, and $D$ is the Tajima statistic. Z-Test: Codon-based test of neutrality for analysis averaging over all sequence pairs. The probability of rejecting the null hypothesis of strict-neutrality (dN $=\mathrm{dS})$ is shown. Values of $\mathrm{P}$ less than 0.05 are considered significant at the $5 \%$ level and are highlighted $(* \mathrm{p}<0.05, * * \mathrm{p}<0.01, * * * \mathrm{p}$ $<0.001$; ns: not significative). The test statistics: $\mathrm{dN}-\mathrm{dS}$, neutrality; $\mathrm{dN}>\mathrm{dS}$ positive selection; $\mathrm{dN}<\mathrm{dS}$ purifying selection are shown. $\mathrm{dS}$ and $\mathrm{dN}$ are the numbers of synonymous and nonsynonymous substitutions per site, respectively. The variance of the difference was computed using the bootstrap method (1000 replicates). Analyses were conducted using the Nei-Gojobori method. All positions containing gaps and missing data were eliminated. 


\section{Table 3 (on next page)}

Genetic heterogeneity and genetic flow of alpA.

Genetic heterogeneity and genetic flow were detected using the following tests: Hst, Kst, Kst*, Z, Z*. The HBK, Snn and chi squared tests were performed using the haplotype frequencies under the permutation of 1000 repetitions, as well as the tests for haplotype diversity of Gst, Nst, Fst. In parenthesis, the gene flow ( $\mathrm{Nm}$ ) value was estimated from FsT using the software DnaSP 5.10. Statistical significance: ${ }^{*} p<0.05$, ${ }^{* *} p<0.01,{ }^{* * *} p<0.001$; ns: not significative. 
Genetic

differentiation

Chi2 Hst $0.003 * *$ $1501.4 \mathrm{~ns}$ Kst 0.090**

Kst *
$0.034^{* * *}$

Z

$4011.8^{* * *}$

$\begin{array}{cc}Z^{*} & \text { Snn } \\ 7.837^{* * *} & 0.316^{* *}\end{array}$
$0.316^{* * *}$

$7.837 * * *$ $0.011(44.82)$

Genetic

flow

Gst
$0.011(44.82)$

GammaST

$0.178(2.30)$
Fst

$0.120(3.63)$ 


\section{Table 4 (on next page)}

Pairwise analysis of differentiation and genetic flow in the populations of alpA.

The FsT and GammaST differentiation parameters are shown. The populations of Colombian isolates are denoted as follows: G: Gastritis, GA: Gastric Adenocarcinoma, IM: Intestinal Metaplasia, IGA: Intestinal Gastric Adenocarcinoma, DGA: Diffuse Gastric Adenocarcinoma, G/DU: Gastritis + Duodenal ulcer, IM/DU: Metaplasia + Duodenal ulcer. A total of 142 sequences were used for the analyses using DnaSP V 5.10. The significance of genetic differentiation was assumed as follow: $<0.05=$ little, $0.05-0.15=$ moderate, $0.15-0.25=$ high and $>0.25=$ complete (Hartl \& Clark, 1997). Significative FsT values are highlighted in bold. 


\begin{tabular}{ccc} 
Populations & & \\
\hline Amerind & FsT & GammaST \\
\hline G vs. hspAmerind & $\mathbf{0 . 2 5 6}$ & $\mathbf{0 . 1 1 3}$ \\
GA vs. hspAmerind & $\mathbf{0 . 3 2 5}$ & $\mathbf{0 . 1 5 1}$ \\
IM vs. hspAmerind & $\mathbf{0 . 2 7 6}$ & $\mathbf{0 . 1 4 1}$ \\
IGA vs. hspAmerind & $\mathbf{0 . 2 8 1}$ & $\mathbf{0 . 2 1 2}$ \\
DGA vs. hspAmerind & $\mathbf{0 . 3 7 9}$ & $\mathbf{0 . 3 2 8}$ \\
G/DU vs. hspAmerind & $\mathbf{0 . 2 4 6}$ & $\mathbf{0 . 2 4 3}$ \\
IM/DU vs. hspAmerind & $\mathbf{0 . 5 0 4}$ & $\mathbf{0 . 3 7 3}$ \\
Europe & & \\
G vs. HpEurope & $\mathbf{0 . 1 2 5}$ & $\mathbf{0 . 1 4 3}$ \\
GA vs. HpEurope & $\mathbf{0 . 1 2 5}$ & $\mathbf{0 . 1 4 8}$ \\
IM vs. HpEurope & $\mathbf{0 . 1 3 1}$ & $\mathbf{0 . 1 5 5}$ \\
IGA vs. HpEurope & $\mathbf{0 . 1 3 3}$ & $\mathbf{0 . 1 6 0}$ \\
DGA vs. HpEurope & $\mathbf{0 . 1 1 9}$ & $\mathbf{0 . 1 2 8}$ \\
G/DU vs. HpEurope & $\mathbf{0 . 1 1}$ & $\mathbf{0 . 1 2 3}$ \\
IM/DU vs. HpEurope & $\mathbf{0 . 1 5 3}$ & 0.093 \\
Asia & & \\
G vs. HpAsia & $\mathbf{0 . 1 1 9}$ & $\mathbf{0 . 1 2 3}$ \\
GA vs. HpAsia & $\mathbf{0 . 1 3 5}$ & $\mathbf{0 . 1 3 7}$ \\
IM vs. HpAsia & $\mathbf{0 . 1 2 3}$ & $\mathbf{0 . 1 3 2}$ \\
IGA vs. HpAsia & $\mathbf{0 . 1 3 2}$ & $\mathbf{0 . 1 3 3}$ \\
DGA vs. HpAsia & $\mathbf{0 . 1 5 3}$ & $\mathbf{0 . 1 1 8}$ \\
G/DU vs. HpAsia & $\mathbf{0 . 1 1}$ & 0.099 \\
IM/DU vs. HpAsia & $\mathbf{0 . 2 0 4}$ & 0.086 \\
Africa & & \\
G vs. HpAfrica & $\mathbf{0 . 1 2 8}$ & $\mathbf{0 . 1 3 4}$ \\
GA vs. HpAfrica & $\mathbf{0 . 1 2 3}$ & $\mathbf{0 . 1 3 7}$ \\
IM vs. HpAfrica & $\mathbf{0 . 1 3 1}$ & $\mathbf{0 . 1 4 8}$ \\
IGA vs. HpAfrica & $\mathbf{0 . 1 2 4}$ & $\mathbf{0 . 1 6 3}$ \\
DGA vs. HpAfrica & $\mathbf{0 . 0 9 9}$ & $\mathbf{0 . 1 3 8}$ \\
\hline /DU vs. HpAfrica & $\mathbf{0 . 1 1 1}$ & $\mathbf{0 . 1 4 5}$ \\
\hline
\end{tabular}

